\title{
The effect of steam inhalation on nasal obstruction in patients with allergic rhinitis
}

\author{
Vannipa Vathanophas, ${ }^{1}$ Piyanuch Pattamakajonpong, ${ }^{2}$ Paraya Assanasen, ${ }^{1}$ Triphoom Suwanwech ${ }^{1}$
}

\begin{abstract}
Background: Nasal obstruction is often impact on quality of life in allergic rhinitis (AR). The steam inhalation is one of widely used home remedies to soothe and open the nasal passages. Furthermore, steam inhalation may provide change in objective nasal airway assessment.
\end{abstract}

Objectives: To compare the effect of steam inhalation on nasal obstruction between patients with AR and normal individuals, as well as the change in the cross-sectional area of the nasal cavity and in nasal airway resistance (NAR) between 2 groups.

Method: A prospective comparative, parallel group study was conducted in AR and normal individuals. Steam with the temperature of $42-44^{\circ} \mathrm{C}$ was inhaled. Variables obtained before and after steam inhalation were compared.

Results: After steam inhalation, nasal symptom score, combined global symptoms, and Visual Analog scale (VAS) of combined global symptoms of AR patients showed statistically significant improvement. Whereas, normal individuals, there was statistically significant improvement only in sneezing and combined global symptoms. Meanwhile, the change of each measurement score, combined global symptoms, and VAS of the symptoms in those with AR were significantly higher than those of normal individuals. Total nasal airflow, NAR, volume, and mean minimal cross-sectional area of AR patients tended to better improve after steam inhalation.

Conclusion: The steam inhalation significantly improved nasal obstruction in AR patients, but no statistical significant difference between both groups for any parameters. The different response between the 2 groups may be due to different nasal mucosa sensitivity to stimuli.

Key words: allergic rhinitis, steam inhalation, nasal obstruction, nasal airway resistance, anterior rhinomanometry

\author{
Affiliations: \\ ${ }^{1}$ Department of Otorhinolaryngology, Faculty of Medicine Siriraj \\ Hospital, Mahidol University, Bangkok, Thailand \\ ${ }^{2}$ Hua Hin Hospital, Prachuap Khiri Khan, Thailand
}

Corresponding author:

Triphoom Suwanwech

E-mail: triphoom.suw@mahidol.ac.th

\section{Introduction}

Allergic rhinitis (AR) is a common chronic respiratory illness with the prevalence of $25 \%$ of the world population. ${ }^{1}$ Bunnag et al., ${ }^{1,2}$ showed that $44.2 \%$ of the Thai population had AR. Despite not being a fatal illness, AR worsens quality of life and can lead to several comorbidities if left untreated. Furthermore, the cost of treatment has been increasing. Hence, other alternative treatments have been searched to reduce such costs.

Symptoms of AR occur after exposure to allergens which stimulates mast cells, leading to certain mediators release such as histamine and tryptase. Its symptoms include nasal obstruction, runny nose, nasal itching and sneezing. Treatments of AR consist of patients' self-care education, pharmacotherapy, allergen immunotherapy, and surgery. ${ }^{3}$

Nowadays, other therapies are searched to be complementary treatments. In particular, steam inhalation has been shown to alleviate nasal obstruction and runny nose in patients with $\mathrm{AR} .{ }^{4}$ Moreover, in AR patients with nasal obstruction, inhaling steam before using nasal spray (i.e. intranasal steroids, intranasal decongestant) can enhance the contact between drug and nasal mucosa, leading to increased effectiveness of treatment. 
Olson and Bende ${ }^{5}$ studied the effect of steam inhalation and cold air on the nasal mucosa of normal individual. Steam inhalation increased nasal mucosal temperature and decreased nasal airway resistance (NAR) whereas cold air breathing increased NAR.

Steam inhalation has been shown to relieve nasal symptoms associated with AR..$^{6-8}$ However, opposite result was reported by Naito et al. ${ }^{9}$ who compared NAR before and after nasal hyperthermia. The NAR significantly increased after nasal hyperthermia. The benefits of steam inhalation in alleviating nasal obstruction in Thai patients with AR and normal Thais have not been studied. In the present study, we aimed to compare the change of nasal symptoms especially nasal obstruction after steam inhalation in patients with AR and normal individuals. We hypothesized that nasal mucosa of those with AR was sensitive to steam inhalation than that of normal individuals.

\section{Study Design}

This observation clinical research case control study was conducted at department of Otolaryngology, Siriraj Hospital, Mahidol University. The study was approved by Siriraj Institution Review Board (SIRB) and granted by Faculty of Medicine, Siriraj Hospital, Mahidol University. Informed consents were obtained from all participants.

\section{Materials and Methods}

A prospective study was conducted to compare the outcomes before and after steam inhalation in patients with AR and normal individuals during October 14, 2008 and November 30, 2009. Inclusion criteria of AR group were those with the age range of 20-60 years who came to the outpatient clinic of Department of Otorhinolaryngology, Siriraj Hospital and had positive skin prick test $(\geq 3 \mathrm{~mm}$ wheal diameter with flare) as well as moderate to severe nasal obstruction (symptom score $\geq 2$ : 0 = no symptom, $1=$ mild symptom, $2=$ moderate symptom, 3 = severe symptom). Besides, patients with AR had to stop taking oral antihistamine, oral decongestant, leukotriene antagonist, $\mathrm{H}_{2}$ blocker for at least 1 week, intranasal medications (i.e. topical decongestant, intranasal corticosteroid) for 2 weeks and systematic steroids for 4 weeks.

For normal individuals, healthy volunteers aged 20-60 years without any nasal symptom or medical problem were recruited. The exclusion criteria included the presence of nasal abnormalities such as severe deviated nasal septum, nasal polyp, nasal tumor, sinus infection or still taking any medications for a specified period of time as well as unwilling to participate in this study.

\section{Sample size calculation}

The objective of study was to compare the minimal cross-sectional area (MCA) at the nasal valve before and after steam inhalation in Thais since the average MCA of normal Thais was $0.55 \pm 0.13$ square centimeters, ${ }^{10}$ for steam inhalation to be effective, MCA should be $0.66 \mathrm{~cm}^{2}$ with standard deviation (SD) of the change of 0.2 (1.5 times of 0.13 ).
Thus, for type I error which was 0.05 with power of 0.8 , at least 28 subjects were needed in the normal group. Since the average MCA in Thai patients with AR was not available, it was assumed that at least 28 people were also required in the AR group. The study design was shown in Figure 1.

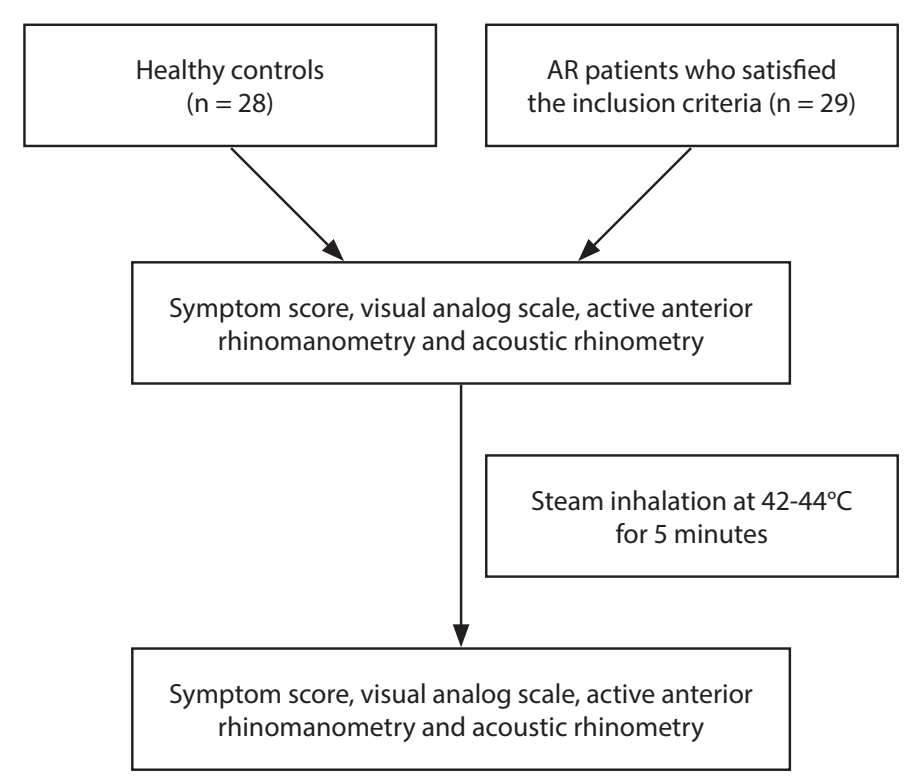

Figure 1. Study design

\section{Experimental protocol}

Demographic data including nasal symptoms were recorded in the case record form and reviewed by investigator. Objective parameters included the assessment of NAR and nasal airflow by active anterior rhinomanometry as well as MCA and nasal volume by acoustic rhinometry. Both active anterior rhinomanometry and acoustic rhinometry were the standard tests to evaluate nasal obstruction objectively.

Patients with AR and normal individuals underwent steam inhalation at $42-44^{\circ} \mathrm{C}$ for 5 minutes with the total follow up duration of 30 minutes. Additionally, the telephone follow-up was done by research assistant on the next day for nasal symptoms or any improvement after steam inhalation.

\section{Subjective measurements}

In this study, the assessment of nasal symptoms was done during two weeks before and immediately after steam inhalation treatment using the four-point rating scale $(0=$ no symptom, 1 = mild symptom, 2 = moderate symptom, 3 = severe symptom). Symptom score was used to assess the severity of symptoms, e.g. runny nose, sneezing, nasal obstruction, nasal itch, postnasal drip, facial pain, headache, itchy eyes, red eyes, watering eyes. Combined global symptom was the summation of nasal and eye symptoms, headache, and facial pain.

Visual analog scale of overall nasal symptoms was the severity assessment of all nasal symptoms on the scale of $10 \mathrm{~cm}$ in length. The $0 \mathrm{~cm}$ indicated no symptom, whereas $10 \mathrm{~cm}$ indicated the most severe symptom. 


\section{Objective measurements}

Active anterior rhinomanometry is the measurement of airflow (total nasal airflow, $\mathrm{cm}^{3} / \mathrm{sec}$ ), and total nasal airway resistance (total NAR, $\mathrm{kPa}$ ) in both left and right nasal cavities. We used ATMOS rhinomanometer 220. Both parameters were reported at 75 Pascals pressure difference. ${ }^{11}$

Acoustic rhinometry is the measurement of the minimal cross-sectional area (MCA) in both nasal cavities (total MCA, $\mathrm{cm}^{2}$ ). It could locate where the MCA was, starting from the anterior nares. It also measured the volume of both nasal cavities (total nasal volume, $\mathrm{cm}^{3}$ ). We used Acoustic rhinometer (model AR-I 1003; Hood laboratories, Pembroke, MA).

\section{Statistical analysis}

Data of patients in each group was analyzed by comparing variables obtained before and after steam inhalation. These variables included nasal symptom scores, combined global symptoms, visual analog scale of overall nasal symptoms, nasal airflow, NAR, MCA, and nasal volume. The net changes of variables obtained before and after steam inhalation were compared between 2 groups.

Since all data were not normally distributed, nonparametric statistics was used for analysis. Wilcoxon-signed rank test was used to compare data obtained before and after steam inhalation within the same group, whereas Mann-Whitney U test was used to compare the data or net change of variables between 2 groups.

The $p$-value $<0.05$ indicated statistical significance. The Statistica ${ }^{\varpi}$ version 5.0 (Stat soft Inc., USA) was used for statistical analysis.

\section{Results}

There were 57 subjects with the age range of 21-54 years ( mean $=36, \mathrm{SD}=10.3)$. They were divided into 2 groups 29 with AR (19 female, 10 male) and 28 normal individuals (14 male, 14 female).

Regarding the net changes of variables in patients with AR before and after steam inhalation, the subjective results showed a statistically significant improvement in every symptom $(p=0.01)$. In addition, there was a statistically significant decrease in the combined global symptoms and the overall nasal symptoms as measured by VAS $(p<0.01)$.

Meanwhile, steam inhalation increased total nasal air flow at $75 \mathrm{~Pa}(13 \%)$, total nasal volume $(9.6 \%)$, and total MCA (41\%) and decreased total NAR at $75 \mathrm{~Pa}(11 \%)$ in patients with AR; however, these changes did not reach statistical significance.

Telephone follow-up of patients with AR on next day after steam inhalation showed that improvement of nasal symptoms after steam inhalation lasted 7.1 hours (from 1 hour to more than 4 hours).

Regarding the net changes of variables of normal individuals before and after steam inhalation, subjective results showed that the majority of variables did not change significantly, except sneezing and combined global symptoms $(p=0.01)$. Steam inhalation increased total nasal airflow (4.5\%), total nasal volume (3.5\%), and total MCA (5.1\%), and decreased total NAR (4.3\%) in normal individuals; however, these change did not reach statistical significance.

By comparing the net changes of variables before and after steam inhalation between patients with $\mathrm{AR}$ and normal individuals, subjective results demonstrated a statistically significant improvement $(p=0.01)$ in almost every variable in patients with AR compared with those in normal individuals, except headache $(p>0.05)$ (Table 1). However, there were no significant improvement in objective variables (total nasal airflow, total nasal volume, total MCA and total NAR) between patients with AR and normal individuals $(p>0.05)$ (Table 1). Nonetheless, no complication was reported in any patient during the study.

Table 1. Comparison of the net changes of variables before and after steam inhalation in patients with allergic rhinitis and normal individuals

\begin{tabular}{|c|c|c|c|}
\hline & \multicolumn{3}{|c|}{ Net change } \\
\hline & $\begin{array}{l}\text { Patients with } \\
\text { allergic rhinitis }\end{array}$ & $\begin{array}{c}\text { Normal } \\
\text { individuals }\end{array}$ & P value ${ }^{\star}$ \\
\hline Rhinorrhea & {$\left[\begin{array}{c}-1 \\
{[(-2)-0]}\end{array}\right.$} & $\begin{array}{c}0 \\
{[0-0]}\end{array}$ & $<0.01$ \\
\hline Itching & {$\left[\begin{array}{c}-1 \\
{[(-2)-0]}\end{array}\right.$} & $\begin{array}{c}0 \\
{[0-0]}\end{array}$ & $<0.01$ \\
\hline Sneezing & {$[(-2)-(-1)]$} & $\begin{array}{c}0 \\
{[(-1)-0]}\end{array}$ & $<0.01$ \\
\hline Nasal blockade & {$\left[\begin{array}{c}-1 \\
{[(-2)-0]}\end{array}\right.$} & $\begin{array}{c}0 \\
{[0-0]}\end{array}$ & $<0.01$ \\
\hline Postnasal drip & $\begin{array}{c}-1 \\
{[(-2)-0]}\end{array}$ & $\begin{array}{c}0 \\
{[0-0]}\end{array}$ & $<0.01$ \\
\hline Facial pain & $\begin{array}{c}0 \\
{[(-1)-0]}\end{array}$ & $\begin{array}{c}0 \\
{[0-0]}\end{array}$ & $<0.01$ \\
\hline Headache & $\begin{array}{c}0 \\
{[(-1)-0]}\end{array}$ & $\begin{array}{c}0 \\
{[0-0]}\end{array}$ & 0.18 \\
\hline Eye symptom & $\begin{array}{c}-1 \\
{[(-1)-0]}\end{array}$ & $\begin{array}{c}0 \\
{[0-0]}\end{array}$ & $<0.01$ \\
\hline $\begin{array}{l}\text { Combined global } \\
\text { symptoms }\end{array}$ & $\begin{array}{c}-8 \\
{[(-10)-(-4)]}\end{array}$ & $\begin{array}{c}0 \\
{[(-1.3)-0]}\end{array}$ & $<0.01$ \\
\hline $\begin{array}{l}\text { Visual analog scale of } \\
\text { global nasal symptoms }\end{array}$ & $\begin{array}{c}-1.3 \\
{[(-3.1)-(-0.8)]}\end{array}$ & $\begin{array}{c}0 \\
{[0-0]}\end{array}$ & $<0.01$ \\
\hline Total nasal air flow & $\begin{array}{c}20 \\
{[(-20)-64]}\end{array}$ & $\begin{array}{c}8 \\
{[(-30)-55]}\end{array}$ & 0.57 \\
\hline Total NAR & $\begin{array}{c}-0.01 \\
{[(-0.04)-0.01]}\end{array}$ & $\begin{array}{c}-0.01 \\
{[(-0.03)-0.03]}\end{array}$ & 0.54 \\
\hline Mean MCA & $\begin{array}{c}0.04 \\
{[(-0.05)-0.1]}\end{array}$ & $\begin{array}{c}0.03 \\
{[(-0.09)-0.2]}\end{array}$ & 0.84 \\
\hline Total nasal volume & $\begin{array}{c}0.3 \\
{[(-0.8)-1.1]}\end{array}$ & $\begin{array}{c}0.6 \\
{[(-0.7)-1.6]}\end{array}$ & 0.26 \\
\hline
\end{tabular}

Note

${ }^{*}$ obtained by Wilcoxon-signed rank test

Median is the representative value of each parameter $\left(25^{\text {th }}-75^{\text {th }}\right.$ percentile).

Net change $=$ value of a variables after steam inhalation - value of a variables before steam inhalation 


\section{Discussion}

Steam inhalation has been widely used to relieve nasal obstruction in patients with AR. Inhalation of hot and humid air increases the nasal mucosal temperature of patients with AR, resulting in inhibition of chemical secretion from mast cell. ${ }^{8}$ In supported of this concept, Georgitis ${ }^{7}$ demonstrated that the histamine level in nasal mucus of patients with AR decreased significantly after steam inhalation, leading to decreased nasal obstruction and vascular leakage. ${ }^{11}$ Steam inhalation was able to quickly relieve cold symptoms by reducing Rhinovirus. ${ }^{12}$ Furthermore, it lowered various inflammatory processes and immunological responses as a result of common cold.

The inhaled steam condenses on nasal mucosa, resulting in increased moisture level with nasal mucosa. ${ }^{13}$ Additionally, it decreases the osmolarity of mucus ${ }^{14}$ so that sticky nasal mucus and phlegm are more easily removed. ${ }^{4}$ Steam also enhances the integrity of nasal mucosa and reduces mucus secretion from various nasal glands. ${ }^{7}$

In our study, the effect of steam inhalation $\left(42-44^{\circ} \mathrm{C}\right)$ on nasal obstruction in Thai population was investigated. There was a significant improvement in nasal obstruction and other symptoms, such as runny nose, nasal itch, sneezing, headache, eye symptoms, facial pain, and postnasal drip in patients with AR after steam inhalation. Variables from objective tests, including total nasal airflow, total NAR, total MCA, total nasal volume, tended to improve after steam inhalation, but these were not statistically significant.

Recently, Yogeetha et al. ${ }^{6}$ studies the effect of environmental temperature on nasal patency of Asian people. This study was conducted in 50 normal Singaporeans in 2 separate rooms in the departmental laboratory where the temperatures were maintained at $30-33^{\circ} \mathrm{C}$ and an air-conditioned room at $18-22^{\circ} \mathrm{C}$, respectively. The increase in NAR and the decrease in nasal patency were noted in the air-conditioned group, compared with the other group. The significant difference was only evident for subjective parameters, not objective parameters.

Ophir et al. ${ }^{8}$ investigated the effect of inhaling warm and humidified air and room temperature air in patients with AR. Inhaled warm and humidified air $\left(42-44^{\circ} \mathrm{C}\right)$ alleviated $A R$ symptoms in $80 \%$ of the patients and improved nasal obstruction (measured objectively by peak nasal inspiratory and expiratory flow meter, as well as rhinohygrometry) by $67 \%$. However, inhaling room-temperature air decreased AR symptoms by $30 \%$, but had no effect on objective parameters of nasal obstruction.

Georgitis ${ }^{7}$ studied the effect of nasal hyperthermia in patients with perennial AR and found that inhaling steam significantly reduced histamine in nasal discharge, which might be due to the suppression of mediators released from mast cells, basophil, neutrophil and eosinophil.

Theoretically, steam inhalation increases moisture in nasal mucosa and decreases the release of histamine and tryptase since heat could suppress the reaction between allergens and mast cells or basophil. In addition, steam helps to stabilize nasal mucosa, and thus reducing nasal mucus production and vascular permeability. ${ }^{4}$ Moreover, steam inhalation prevented nasal itching, sneezing, and nasal obstruction from allergen challenge.
The disagreement between changes of subjective and objective parameters has been shown in many studies such as Kim et al. ${ }^{14}$ and Yoogetha et al. ${ }^{6}$ In these studies, nasal symptoms, not NAR, were closely related to temperature changes. The relationship between temperature and nasal symptoms was obvious when subjects inhaled cold air, based on a hypothesis that there were temperature receptors on nasal mucosa. ${ }^{5}$ However, no significant change in objective parameters after steam inhalation in our study was probably due to insufficient number of participants with AR. Thus, increased number of participants with AR could lead to more obvious changes in objective parameters.

For normal individuals, steam inhalation improved sneezing and combined global symptoms. The objective parameters showed a similar tendency, but did not reach statistical significance since normal individuals had no nasal symptoms, so the effects of steam inhalation were probably less obvious, or the number of normal participants could be insufficient.

The improvement of objective parameters of nasal obstruction in both groups could be explained by the physiologic responses of nasal mucosa to environment stimuli. In normal conditions, nasal mucosa warms and humidifies air before entering lungs. After cold air inhalation, nasal mucosa needs to warm and humidify air by increasing mucosal surface (blood pooling in arteriovenous anastomosis and cavernous sinusoids), leading to nasal obstruction in some individuals. Conversely, after steam inhalation, nasal mucosa does not need to warm and humidify air, resulting in nasal decongestion. ${ }^{4}$ Hence, steam inhalation is beneficial in those with AR and normal individuals with nasal symptoms caused by common cold.

The improvement of subjective and objective parameters after steam inhalation in patients with AR were more obvious than those in normal individuals. This can be explained by the differences of nasal mucosal sensitivity to steam between 2 groups. Nasal mucosa of those with AR had higher sensitivity to stimuli and irritants compared with that of normal individuals. $^{16}$

In addition to pure steam, some additives such as Tincture benzoin, Thai herbs (lemongrass, ginger) are put into hot water to better improve nasal symptoms. Nonetheless, further studies are needed to investigate the effects of such additives on nasal symptoms (esp. nasal obstruction).

\section{Conclusion}

Steam inhalation is one of the most effective treatments of nasal obstruction in patients with AR. It can also relieve nasal symptoms such as itching, sneezing, runny nose, headache, facial pain, and postnasal drip. Despite no significant changes in objective parameters, the improvement of patients' nasal symptoms was shown in our study and should be the main concern for both patients and physicians. Additionally, steam inhalation can be applied with other pharmacological treatments without any complication or additional costs. 


\section{Acknowledgements}

The author would like to thank Miss Jeerapa Kerdnoppakhun for data management and preparation of this manuscript.

\section{References}

1. Bunnag C. Update management in allergic rhinitis. Siriraj Med J. 2006;58: 622-3.

2. Bunnag C. A survey of allergic rhinitis in Thais. Siriraj Med J. 1995;47: 1027-31.

3. Sheikh J, Jean T. Allergic Rhinitis [Internet]. New York: Medscape; 2017 [cited 2017 Jan 31]. Available from:http://emedicine.medscape.com/article /134825-overview

4. Jareonchasri P. Steam Inhalation and Aromatherapy for Rhinosinusitis. Rhinosinusitis. Bangkok: Pentagon Advertising Limited Partnership; 2010.

5. Olsson P, Bende M. Influence of environmental temperature on human nasal mucosa. Ann Otol Rhinol Laryngol. 1985;94(2 Pt 1):153-5.

6. Yogeetha R, Raman R, Quek KF. Effects of temperature changes on nasal patency. Singapore Med J. 2007;48:304-6.

7. Georgitis JW. Nasal hyperthermia and simple irrigation for perennial rhinitis. Changes in inflammatory mediators. Chest. 1994;106:1487-92.
8. Ophir D, Elad Y, Dolev Z, Geller-Bernstein C. Effects of inhaled humidified warm air on nasal patency and nasal symptoms in allergic rhinitis. Ann Allergy. 1988;60:239-42.

9. Naito K, Miyata S, Baba R, Mamiya T, Senoh Y, Iwata S, et al. The alteration of nasal resistance before and after local exposure to heated aerosol in perennial allergic rhinitis. Rhinology. 1999;37:66-8.

10. Jareoncharsri P, Bunnag C, Voraprayoons S, Dachpunpour P, Ungkanont $\mathrm{K}$, Assanasen P. Acoustic rhinometry of the Thais nose. 5th ed. Bangkok Thailand: ARSR; 2000.

11. Salman SD, Proctor DF, Swift DL, Eveering SA. Nasal resistance: description of a method and effect of temperature and humidity changes. Ann Otol Rhinol Laryngol. 1971;80:736-43.

12. Lwoff A. Death and transfiguration of a problem. Bacteriol Rev. 1969;33: 390-403.

13. Rouadi P, Baroody FM, Abbott D, Naureckas E, Solway J, Naclerio RM. A technique to measure the ability of the human nose to warm and humidify air. J Appl Physiol (1985). 1999;87:400-6.

14. Kim CS, Moon BK, Jung DH, Min YG. Correlation between nasal obstruction symptoms and objective parameters of acoustic rhinometry and rhinomanometry. Auris Nasus Larynx. 1998;25:45-8.

15. Asakura K, Enomoto K, Ara H, Azuma E, Kataura A. Nasal responsiveness to methacholine stimulation in allergic rhinitis patients. Arch Otorhinolaryngol. 1984;239:273-8. 